\begin{abstract}
The Luminosity Functions of Galactic Star Clusters.* Sidney van den Bergh and David Sher, David Dunlap Observatory.-The luminosity functions of 20 galactic clusters down to $m_{p g} \approx 20$ have been determined by means of star counts on 170 plates obtained with the Palomar 48-inch Schmidt. It is found that striking differences exist between the main-sequence luminosity functions of individual clusters. Also it appears that the faint ends of the luminosity functions of galactic clusters differ systematically from the van Rhijn-Luyten luminosity function for field stars in the vicinity of the sun in the sense that (with one exception) all the clusters which were investigated had luminosity

functions which either decrease or remain constant below $M_{p g}=+5$. The differences between individual clusters and the differences between the luminosity functions of clusters on the one hand and field stars on the other show that the luminosity function of star creation is not unique. This result is taken to indicate that the luminosity function with which stars are created probably depends on the physical conditions prevailing in the region of star formation.

\footnotetext{
* This abstract of a paper presented at the 106th Meeting
} of the American Astronomical Society was omitted, through an editorial oversight, from the abstracts of the meeting published in the November 1960 No. 1284 issue of the Astronomical Journal.
\end{abstract}

\title{
NOTICE
}

\section{Graduate Laboratory Development Program}

$T$ HE National Science Foundation announces that March 1, 1961 is the next closing date for receipt of proposals in the Graduate Laboratory Development Program. Proposals received after March 1 will be reviewed following the next closing date, September 1, 1961. This program requires at least $50 \%$ participation by the institution with funds derived from non-Federal sources.

Purpose of the grants is to aid institutions of higher education in modernizing, renovating, or ex- panding graduate-level basic research laboratories used by staff members and graduate students. Only those departments having an on-going graduate training program leading to the doctoral degree in science at the time of proposal submission are eligible at present.

Proposals, as well as requests for additional information, should be addressed to: Office of Institutional Programs, National Science Foundation, Washington 25, D. C.

\section{ERRATUM}

Astron. J. 65, 354.

Left column, line -8 : for 900 read 800 .

Left column, line -5 : for IV read III-IV.

Right column: delete last 11 lines; substitute "The maximum in the space velocity frequency distribution for the thirty weak $C N$ stars occurs at approximately $50 \mathrm{~km} / \mathrm{sec}$, with only $20 \%$ of the velocities exceeding $65 \mathrm{~km} / \mathrm{sec}$." 